\title{
Special Issue "New Perspectives in Intelligent Transportation Systems and Mobile Communications towards a Smart Cities Context"
}

\author{
Giovanni Pau ${ }^{1, *(\mathbb{D})}$, Alessandro Severino ${ }^{2}$ and Antonino Canale ${ }^{1}(\mathbb{D}$ \\ 1 Faculty of Engineering and Architecture, Kore University of Enna, 94100 Enna, Italy; \\ antonino.canale@unikore.it \\ 2 Department of Civil Engineering and Architecture, University of Catania, 95123 Catania, Italy; \\ severinoalessandro@yahoo.it \\ * Correspondence: giovanni.pau@unikore.it
}

Received: 8 October 2019; Accepted: 24 October 2019; Published: 28 October 2019

\begin{abstract}
Intelligent transportation solutions and smart information and communication technologies will be the core of future smart cities. For this purpose, these topics have captivated noteworthy interest in the investigation and construction of cleverer communication protocols or the application of artificial intelligence in the connection of in-vehicle devices by wireless networks, and in in-vehicle services for autonomous driving using high-precision positioning and sensing systems. This special issue has focused on the collection of high-quality papers aimed at solving open technical problems and challenges typical of mobile communications for Intelligent Transportation Systems.
\end{abstract}

Keywords: information and communication technologies for transportation systems; driver and traveler support systems; renewable-energy-based vehicles for green transportation; traffic operations management and control; trust, security, and privacy in transportation networks

\section{Introduction}

Future cities can be identified as "smart" if they will be based on intelligent transportation solutions and smart information and communication technologies. For this reason, these themes have captivated noteworthy interest in the investigation and development of smarter communication protocols. As a consequence, in contemporary years, essential improvements in intelligent transportation systems (ITSs) have been achieved in the growth of in-vehicle systems by the employment of artificial intelligence, in the connection of in-vehicle devices by wireless networks, and in in-vehicle services for autonomous driving using high-precision positioning and sensing systems. In addition, there has also been a significant enhancement of mobile, ad-hoc, and sensor networks and ubiquitous services in the area of mobile communications. For all of these reasons, a thorough synergy between ITS solutions and mobile communications can concretely inaugurate new outlooks for future smart cities.

This special issue has requested the submission of high-quality papers that intend to resolve issues and challenges related to mobile communications for ITS.

\section{A Short Review of the Contributions in This Special Issue}

The authors of [1] perform a thorough investigation of several notable position-based routing protocols designed for vehicle-to-vehicle communication, highlighting their operating mode and limitations. Moreover, the authors present an overview of vehicular ad hoc network (VANET) architecture and a qualitative comparative examination of routing protocols mentioned above. 
The parameters of the comparison are the mobility, traffic density, and forwarding technique, i.e., crucial features for VANETs.

A programming prototype for demand-responsive feeder transportation services to designate vehicles placed at various stations, aimed at picking up passengers at the demand points and carrying them to the rail station is proposed in [2]. The main aim is to reveal the relationship between the total mileage and passenger satisfaction. The methodology proposed by the authors can allow an interactive method for planning feeder transit routing and leading the passenger to determine a proper boarding time window. The usefulness and applicability of the suggested approach are demonstrated with a real-world application, and the obtained results show that the total mileage is significantly lessened, while the total satisfaction is concretely improved, compared with other solutions in the literature.

The authors of [3] suggest a solution in which the employment of fuzzy logic control theory for primary diesel engine speed control is associated with particle swarm optimization to achieve the optimal arrangement of the membership functions. The obtained results are promising, but the use of the proposed method also has some drawbacks since it is complicated and requires a high degree of operators.

The main aim of the platform introduced in [4] is to combine techniques to determine the measure of carbon dioxide released by vehicles, considering the amount produced during combustion in the vehicle's engine. The authors perform examinations and assessments that can be useful when designing and maintaining modern cities. The results obtained in the assessment of the platform introduced by the authors show that the developed solution can be beneficial for traffic control systems in cities since regions with a larger congregation of pollution typically include crossings, traffic lights, and congested areas.

The authors of [5] propose a support system for ship navigation in the fairway. The proposed solution can control the ship automatically by adjusting the rudder to match programmed routes. Then, the ship's acceleration is decreased step by step to reach the berth area at a low speed and, finally, the ship's heading is appropriately regulated by the bow thruster before switching the control method to the automatic berthing system. Numerical simulations are carried out with a training ship model to prove the effectiveness of this proposed system, and the acquired outcomes are promising.

Author Contributions: The authors contributed equally to this work.

Acknowledgments: The authors are grateful to the MDPI for the invitation to act as guest editors of this special issue and want to thank the editorial staff of Future Internet for the kind cooperation, patience, and committed engagement. This special issue was related to the D.D. 407 of 27 February 2018 "AIM-Attrazione e Mobilità Internazionale" issued by the Italian Ministry of Education, University, and Research in implementation of Action I.2 "Mobilità dei Ricercatori" Asse I- PON R\&I 2014-2020, taking into account the written amendment procedure of the PON R\&I 2014-2020, pursuant to articles 30 and 90 of Regulation (EU) 1303/2013 started on 21 February 2018 as well as the relevant implementation regulations.

Conflicts of Interest: The authors declare no conflict of interest.

\section{References}

1. Abbasi, I.A.; Shahid Khan, A. A Review of Vehicle to Vehicle Communication Protocols for VANETs in the Urban Environment. Future Internet 2018, 10, 14. [CrossRef]

2. Sun, B.; Wei, M.; Zhu, S. Optimal Design of Demand-Responsive Feeder Transit Services with Passengers's Multiple Time Windows and Satisfaction. Future Internet 2018, 10, 30. [CrossRef]

3. Tran, T.A. The Optimization of Marine Diesel Engine Rotational Speed Control Process by Fuzzy Logic Control Based on Particle Swarm Optimization Algorithm. Future Internet 2018, 10, 99. [CrossRef] 
4. Silva, M.; Signoretti, G.; Oliveira, J.; Silva, I.; Costa, D.G. A Crowdsensing Platform for Monitoring of Vehicular Emissions: A Smart City Perspective. Future Internet 2019, 11, 13. [CrossRef]

5. Nguyen, V.S. Research on a Support System for Automatic Ship Navigation in Fairway. Future Internet 2019, 11, 38. [CrossRef]

(c)

(C) 2019 by the authors. Licensee MDPI, Basel, Switzerland. This article is an open access article distributed under the terms and conditions of the Creative Commons Attribution (CC BY) license (http://creativecommons.org/licenses/by/4.0/). 